\title{
6. Cardiopatías en la mujer
}

\subsection{Manejo y desenlaces en mujeres con infarto agudo de miocardio con elevación del segmento ST}

Pimentel Morales Gela, Malváez López Héctor Miguel,

Estrada Gallegos Joel, Arizmendi Uribe Efraín,

Mayorquín Carranza Javier, Saturno Chiu Guillermo

Centro Médico Nacional Siglo XXI, IMSS.

Tipo de estudio: Registros locales o nacionales

Introducción: La ICP primaria es el método de reperfusión de elección en pacientes con IAMCEST; sin embargo, este procedimiento conlleva riesgos como embolismo distal o fenómeno de no flujo, los cuales pueden influir en los desenlaces posteriores. Los medicamentos actuales y la nueva tecnología en los diseños de los stents reducen dichas complicaciones. Hay pocos estudios que lo evalúen en mujeres. Objetivo: Evaluar los desenlaces a corto y largo plazo de las mujeres sometidas a ICP primaria en el contexto de IAMCEST en nuestro centro. Metodología: Se evaluaron un total de 124 mujeres sometidas a ICP primaria con diagnóstico de IAMCEST tratadas en nuestro centro. Resultados: Se evaluó un total de 124 mujeres, documentando una edad promedio de $68.9 \pm 10.2$ años, DM2 54\%, HAS 76.6\%, hipercolesterolemia 45.1\%, infartos previos $7.2 \%$, ICP previas $3.2 \%$, CRVC $0.8 \%$, enfermedad multivaso $38.3 \%$. Número de stents implantados $1.3 \pm 0.6$, tromboaspiración en $38.3 \%$, FEVI promedio $42.5 \pm 1.3 \%$, uso de GPIIb/IIla $20 \%$. Se obtuvo un flujo TIMI $381.6 \%$. El seguimiento promedio fue de 30 \pm 11 meses, con 24 defunciones (19\%), revascularización del vaso diana $6.9 \%$, otro vaso $32.2 \%$, reinfarto en $6.9 \%$, trombosis del stent $2.2 \%$, clase funcional I $32.2 \%$, II 8\%, III 0.8\%, IV 0.8\%. Discusión y conclusiones: La ICP primaria en mujeres con IAMCEST no afecta negativamente los desenlaces clínicos cuando el procedimiento lo realizan operadores entrenados, dando mejores resultados, desenlaces y supervivencia. 